Article

\title{
Better Estimated IEM Input Parameters Using Random Fractal Geometry Applied on Multi-Frequency SAR Data
}

\author{
Ali Ghafouri ${ }^{1}$, Jalal Amini ${ }^{1, *}$, Mojtaba Dehmollaian ${ }^{2}$ and Mohammad Ali Kavoosi ${ }^{3}$ \\ 1 School of Surveying and Geospatial Engineering, Faculty of Engineering, University of Tehran, \\ Tehran 1439957131, Iran; ali.ghafouri@ut.ac.ir \\ 2 Center of Excellence on Applied Electromagnetic Systems, School of Electrical and Computer Engineering, \\ University of Tehran, Tehran 1439957131, Iran; m.dehmollaian@ut.ac.ir \\ 3 Department of Geology, Exploration Directorate of National Iranian Oil Company, Tehran 1994814695, Iran; \\ m.kavoosi@niocexp.ir \\ * Correspondence: jamini@ut.ac.ir; Tel.: +98-912-459-4685; Fax: +98-21-8860-4534
}

Academic Editors: Timo Balz, Uwe Soergel, Mattia Crespi, Batuhan Osmanoglu, Nicolas Baghdadi and Prasad Thenkabail

Received: 26 February 2017; Accepted: 4 May 2017; Published: 5 May 2017

\begin{abstract}
Microwave remote sensing can measure surface geometry. Via the processing of the Synthetic Aperture Radar (SAR) data, the earth surface geometric parameters can be provided for geoscientific studies, especially in geological mapping. For this purpose, it is necessary to model the surface roughness against microwave signal backscattering. Of the available models, the Integral Equation Model (IEM) for co-polarized data has been the most frequently used model. Therefore, by the processing of the SAR data using this model, the surface geometry can be studied. In the IEM, the surface roughness geometry is calculable via the height statistical parameter, the rms-height. However, this parameter is not capable enough to represent surface morphology, since it only measures the surface roughness in the vertical direction, while the roughness dispersion on the surface is not included. In this paper, using the random fractal geometry capability, via the implementation of the power-law roughness spectrum, the precision and correctness of the surface roughness estimation has been improved by up to $10 \%$. Therefore, the random fractal geometry is implemented through the calculation of the input geometric parameters of the IEM using the power-law surface spectrum and the spectral slope. In this paper, the in situ roughness measurement data, as well as SAR images at frequencies of $\mathrm{L}, \mathrm{C}$, and $\mathrm{X}$, have been used to implement and evaluate the proposed method. Surface roughness, according to the operational frequencies, exhibits a fractal or a diffractal behavior.
\end{abstract}

Keywords: Synthetic Aperture Radar (SAR); Integral Equation Model (IEM); random fractal geometry

\section{Introduction}

Studying the surface roughness geometry, especially studying the natural surfaces, requires appropriate satellite data and processing methodologies [1-3]. The Synthetic Aperture Radar (SAR) data acquired by the airborne and space-borne sensors has made it possible to examine the surface roughness, which provides useful information for geoscientists and geologists. The backscattered signal in all polarizations is affected by surface roughness, and contains the surface geometry information [4-6]. To measure the surface geometry using the SAR data, the surface geometric parameter(s) must be modeled against the backscattering coefficient on each polarization. Generally speaking, to describe and differentiate the patterns and the geometric surface texture, it is indispensable to model the interaction between the backscattered signals and the surface properties. The radar signal 
is sensitive to certain surface roughness measures, which is determined by the operating radar signal frequency [7-9].

Of the available models, the standard theoretical models of backscattering include: (1) the Kirchhoff Approximation (KA), which encompasses Geometric Optics (GO) and Physical Optics (PO); and (2) the Small Perturbation Model (SPM) [7,10].

The Geometric Optics model for very rough surfaces, the Physical Optics model for moderate roughness, and the Small Perturbation Model for approximately smooth surfaces, have been used. Fung et al. (1992) have developed the Integral Equation Model (IEM) as a physically-based electromagnetic transfer model which combines the Kirchhoff models and the SPM, and which constructs a more applicable model that can theoretically tolerate a really wide range of roughness dimensions. It is of note that the IEM is not restricted to any special frequency range or roughness measures [11].

The IEM exploits the rms-height parameter to characterize the surface geometry [12]. In this paper, the statistical rms-height and the Gaussian auto-correlation function (ACF) have been implemented as the conventional IEM. In the conventional method, the surface roughness geometry is only considered in the vertical direction, and only the Euclidean geometry is applied.

However, using the so called fractal IEM, which considers roughness in the horizontal direction and its dispersion on the surface, it can be possible to improve the surface morphology estimation and soar the precision of the microwave discrimination by means of the power-law surface spectrum and the spectral slope parameter [13].

In this paper, as depicted in Figure 1, to provide the surface geometric characteristics for the IEM computation, the in situ surface roughness measurement has been performed by the field surveying operation. The dielectric constants have been extracted from the tables produced by Martinez et al. (2000) [14]. Furthermore, the SAR satellite data of ALOS PALSAR, Sentinel-C, and TerraSAR, respectively, in the bands $\mathrm{L}, \mathrm{C}$, and $\mathrm{X}$, have been used to compute the surface roughness, i.e., the geological morphology.

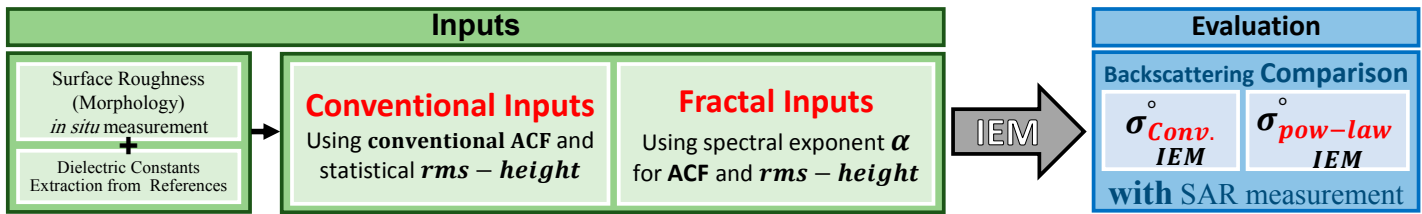

(a)

\begin{tabular}{|c|c|c|c|c|}
\hline \multicolumn{2}{|c|}{ Inputs } & \multirow{4}{*}{ Inverse IEM } & \multicolumn{2}{|c|}{ Evaluation } \\
\hline \multirow{3}{*}{$\begin{array}{c}\text { Backscattering Coefficient }\left(\boldsymbol{\sigma}^{\circ}\right) \\
\text { Calculation using } \\
\text { SAR Satellite measurements }\end{array}$} & L-band : ALOS PALSAR & & \multicolumn{2}{|c|}{ Roughness Comparison } \\
\hline & C-band : Sentinel-1A/SAR-C & & $\boldsymbol{S}_{\text {Conv }}$ & $\begin{array}{c}\boldsymbol{S}_{\text {pow-law }} \\
\text { IEM }\end{array}$ \\
\hline & X-band : TerrsaSAR-X & & \multicolumn{2}{|c|}{ with Field measurement } \\
\hline
\end{tabular}

(b)

Figure 1. Flowchart of the study and results evaluation. (a) Direct model evaluation: computation of backscattering using the in situ measured data; (b) inverse model evaluation: calculation of surface roughness using the backscattering coefficient.

In this paper, the IEM geometric input parameters have been calculated using three different methods for two types of surface assumptions; two conventional geometry methods (stationary surface assumption) and the random fractal geometry (for power-law surface assumption). After running the model with each of the inputs, the backscattering coefficient at each point corresponding to each pixel of the SAR measurement data is computed (Figure 1a). In addition, through the IEM inversion method, the surface parameters are calculated, corresponding to the in situ measurements (Figure 1b). The IEM computation results, as well as the inverse IEM compared with the measurements, can show the model's level of efficacy. 
The IEM calculated and the SAR measured backscattering coefficients are mutually compared to evaluate the methodology (Figure 1a). Additionally, comparing the in situ rms-height with the inverse IEM results, the level of efficacy when using the power-law inputs versus the conventional ones can be cleared [12,15] (Figure 1b).

In Section 2, the IEM backscattering model with the conventional inputs will be addressed. The methodology of random fractal geometry will be developed in Section 3, in order to determine the power-law surface parameters as the input of the IEM. Finally, the implementation and validation of the fractal IEM will be discussed in Section 4 for the multi-frequency SAR data. Section 5 presents the conclusion.

\section{The IEM Backscattering Model}

Backscattering is the amount of the scattered signal per surface unit in the scattering angle from 0 to 180 degrees [16]. The calculation of the backscattering coefficient $\left(\sigma^{\circ}\right)$ process depends on the satellite antenna specifications. As defined by Fung et al. (1992) and explained by themselves (1994), the IEM model describes the relation of the backscattering coefficient to the surface roughness parameters, as well as the dielectric constant and the incidence angle. The IEM is characterized for the co-polarized and cross-polarized backscattering calculations $[8,17]$. Yet, in this paper, just the co-polarized equation is implemented. The co-polarized backscattering coefficient equation according to Fung et al. (2004), which is termed I²EM by Ulaby (2014), is as follows $[7,8,10,11,16]$ :

$$
\sigma_{p p}^{\circ}=\frac{k^{2}}{4 \pi} e^{-2 k^{2} s^{2} \cos ^{2} \theta} \sum_{n=1}^{+\infty}\left|I_{p p}^{n}\right|^{2} \frac{W^{(n)}(2 k \sin \theta, 0)}{n !}
$$

where:

$$
I_{p p}^{n}=(2 k \mathrm{~s} \cos \theta) f_{p p} \exp \left(-k^{2} \mathrm{~s}^{2} \cos ^{2} \theta\right)+(k \mathrm{~s} \cos \theta)^{n} F_{p p}
$$

and $p p$ is either the $h h$ or $v v$ polarizations; $k$ stands for the radar wavenumber ( $k=\frac{2 \pi}{\lambda}, \lambda$ wavelength); $s$ is the rms-height; $\theta$ denotes the incidence angle; and $W^{(n)}$ is the Fourier transform of $n$th power of the ACF. $f_{h h}, f_{v v}, F_{h h}$, and $F_{v v}$ are approximated by the following equations [10,16]:

$$
\begin{gathered}
f_{h h}=\frac{-2 R_{h}}{\cos \theta} \\
f_{v v}=\frac{2 R_{v}}{\cos \theta} \\
F_{h h}=2 \frac{\sin ^{2} \theta}{\cos \theta}\left[4 R_{h}-\left(1-\frac{1}{\varepsilon}\right)\left(1+R_{h}\right)^{2}\right] \\
F_{v v}=2 \frac{\sin ^{2} \theta}{\cos \theta}\left[\left(1-\frac{\varepsilon \cos ^{2} \theta}{\varepsilon-\sin ^{2} \theta}\right)\left(1-R_{v}\right)^{2}-\left(1-\frac{1}{\varepsilon}\right)\left(1+R_{v}\right)^{2}\right]
\end{gathered}
$$

where the horizontally and vertically polarized Fresnel reflection coefficients, $R_{h}$ and $R_{v}$, are given by the following:

$$
\begin{aligned}
& R_{h}=\frac{\cos \theta-\sqrt{\varepsilon-\sin ^{2} \theta}}{\cos \theta+\sqrt{\varepsilon-\sin ^{2} \theta}} \\
& R_{v}=\frac{\varepsilon \cos \theta-\sqrt{\varepsilon-\sin ^{2} \theta}}{\varepsilon \cos \theta+\sqrt{\varepsilon-\sin ^{2} \theta}}
\end{aligned}
$$

where $\varepsilon$ is the ground relative dielectric constant; a complex number concerns the storage and dissipation of electricity.

In the IEM, $s$ and the surface ACF are the two geometric elements whose calculations are the main issue considered in this paper. 
Due to the nonlinearity of (1), the model inversion, i.e., solving the IEM for its parameters analytically, is almost impossible. There are a few arithmetic methods which can be employed to calculate the surface parameters knowing the backscattering coefficient and the imaging parameters [12]. In spite of the development of intelligent computation methods such as neural networks and the Bayesian method, one of the best and most direct methods in this respect is the Look-up table (LUT) [18,19]. In the LUT method, the backscattering coefficient values for different values of the surface roughness parameters and dielectric constant are calculated using (1), and then, the surface parameters corresponding to the backscattering coefficients can be calculated by interpolation and reversed matching.

Section 2.1 describes the validity range and Section 2.2 exhibits the conventional input parameters calculation of this model.

\subsection{The IEM Validity Region}

The IEM is applicable on a wide range of surfaces, from smooth to rough. The IEM's validity range, given by Fung et al. (1994), is:

$$
\begin{gathered}
k s<3 \\
(k l)(k s)<\mu \sqrt{|\varepsilon|} \\
\cos ^{2} \theta \frac{(k s)^{2}}{\sqrt{0.46 k l}} \exp [-\sqrt{2 \times 0.46 k l(1-\sin \theta)}] \ll 1
\end{gathered}
$$

where $\mu$ is a constant and its Gaussian value, and the exponential ACFs are 1.6 and 1.2, respectively $[10,20]$.

Dierking (1999) has also estimated the validity range of the IEM for different frequencies, similar to the ones specified by Fung et al. [21]. Furthermore, different studies have concerned the real validity range of this model and it is mostly shown that the applicable validity domain of this model is more limited $[18,22,23]$.

\subsection{Conventional Inputs for IEM}

The rms-height is calculated using the following formula (6):

$$
s=\sqrt{\frac{1}{N}\left[\left(\sum_{i=1}^{N} z_{i}^{2}\right)-N \bar{z}^{2}\right]} \forall \bar{z}=\frac{1}{N} \sum_{i=1}^{N} z_{i}
$$

Moreover, $W^{(n)}$ is another geometric term in the IEM, i.e., the Fourier transform of the $n$th power of the surface ACF, $A(x)$, either its exponential, or Gaussian regression [24,25] (i.e., respectively (7)-(9)):

$$
\begin{gathered}
A(x)=\frac{\sum_{i=1}^{N-j} z_{i} z_{i+j}}{\sum_{i=1}^{N} z_{i}^{2}} \\
A(x)=e^{\frac{-|x|}{l}} \\
A(x)=e^{\frac{-x^{2}}{l^{2}}}
\end{gathered}
$$

where $l$ is the correlation length. Because of its dependence on the profile length, as well as the rms-height, the calculation of the correlation length has been considered as a difficult problem $[9,26]$. In this paper, both exponential and Gaussian ACFs are used for conventional implementation, which have presented a better estimation and have been frequently recommended $[3,12,15]$. In the calculation of the ACFs, the correlation length is considered as one third of the semivariogram range [27]. 


\section{Power-Law Inputs for the IEM}

Functions, like $y$, proportional to some power of the input $x$ (i.e., $y=x^{p}$ ), are power-law functions. Power-law surfaces have the spectrum in the form of $S(f)=c / f^{\alpha}$ ( $\alpha$ : spectral slope). In natural surface modeling, unlike the conventional geometry, the random fractal geometry provides better results. The term "fractal", in many cases, is considered as the same as "self-similar". Natural phenomena are statistically self-similar; i.e., every part of their structure has statistical properties (the mean and the standard deviation) similar to those of the whole structure [28].

The surfaces with a power-law roughness spectrum over the interval $0 \leq f \leq \infty$ are ideal random fractals. For such surfaces, the rms-height $s$, the correlation length $l$, and the ACF do not exist [21,29] Thus, the surface modeling must be realized within the limits of the spatial frequencies (i.e., sampling rates) $f_{\min } \leq f \leq f_{\max }$. This is a semi-self-affine surface [13,30]. In other words, using the fractal geometry, natural surfaces can be modeled through the power-law form within $f_{\min }$ and $f_{\max }$.

Yordanov et al. (2002) present a general expression for the ACF valid for the arbitrary topological dimension [31]. Since after the roughness measurement of the study sites, the geometric parameters are considered to be calculated on some arbitrary linear profiles, the ACF for a linear profile of the samples can be realised in the form of [32]:

$$
A(x)=2 \pi^{D-1} \int_{k_{\min }}^{k_{\max }} k^{D-1-\alpha} \cos (k x) d k
$$

where $k$ is the wavenumber and $\alpha$ has a limited amount; $D<\alpha<D+2, D$ is the topological dimension, and for the linear profile, it is considered one $(D=1)[29,33]$, representing the slope of the linear best-fit of the power spectral density (PSD) on a logarithmic scale [29,32].

It is noted that this linear best-fit must be applied to the trendless profile. In other words, the spectral slope $(\alpha)$ might not include the trend part of the PSD, the early part of the PSD [29].

According to Dierking (1999), in addition to the ACF, two other regular geometric surface parameters, the power-law rms-height and the correlation length for a linear profile, can also be calculated using the spectral slope parameter [21]:

$$
\begin{gathered}
s=\sqrt{c L^{\alpha-1} /(\alpha-1)} \\
l=\frac{(\alpha-1)^{2} L}{2(2 \alpha-1)}
\end{gathered}
$$

where $L$ is the profile length, and $c$ is the spectral constant or the spectral offset [21].

Hence, in order to evaluate the role of the random fractals for improving the IEM results, having ascertained the surface morphology, using (8) and (9), the ACF for conventional- 1 and conventional-2 methods, respectively, is achieved. The rms-height (s) can be calculated using (6) for these two conventional methods.

For the power-law calculation of the input parameters, the welch method is applied to the in situ measured data to calculate the spectral slope, $\alpha$. Having obtained $\alpha$ and using (11), the rms-height (s) is computed. Using (10), the ACF is computed.

\section{Implementation and Evaluation}

Three different methods for the calculation of the input parameters of the IEM are implemented for the in situ field measurement to apply the methodology of the flowchart (Figure 1a). Afterward, following the second flowchart (Figure 1b), the LUT can be formed to determine the surface roughness of the terrestrial points corresponding to each measured SAR backscattering (on L, C, and X-bands) pixels. The case study is located in western Iran, between the cities of Ilam and Dehloran. From the view point of geology, this region is known as the "Anaran" anticline, located in the Zagros fold-thrust belt. Figure 2 depicts the geographic location of the case study and the 10 measurement sites. Figure $2 \mathrm{~b}$ 
depicts the main geological formations of the region; however, the morphology of two of them (Gurpi Fm and Quaternary Sediments) is not considered to have been studied in this paper.

The field measurement is planned in the form of a mesh of points; thus, the geometric parameters for any arbitrary profiles on the surface can be calculated. The in situ surface roughness measurement is performed on ten different sites with different morphologies using the programmable surveying Total Station (Trimble ${ }^{\mathrm{TM}} 5600$ ). This instrument is used to measure a $25 \times 25$ mesh with a $50 \mathrm{~cm}$ interval on each measurement site. The measurement instrument has a better than one centimeter precision. Being measured as a mesh of points allows for the calculation of roughness parameters along any arbitrary profile and certainly increases the precision of the parameters estimation. Generally, there are three formations belonging to different geological periods. Table 1 shows the details of these geological formations.

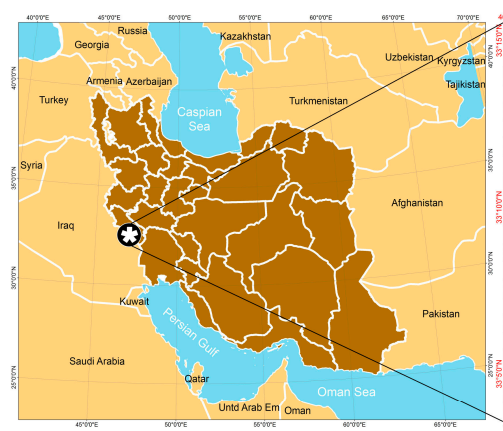

(a)

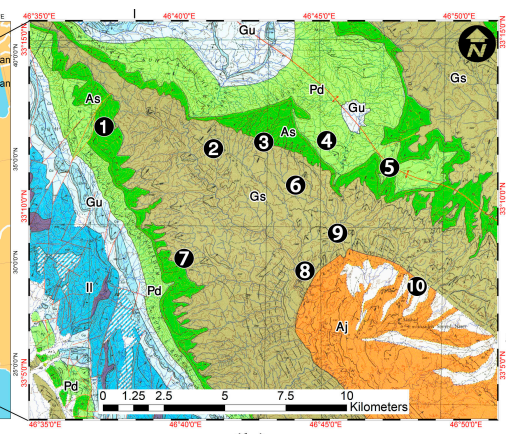

(b)

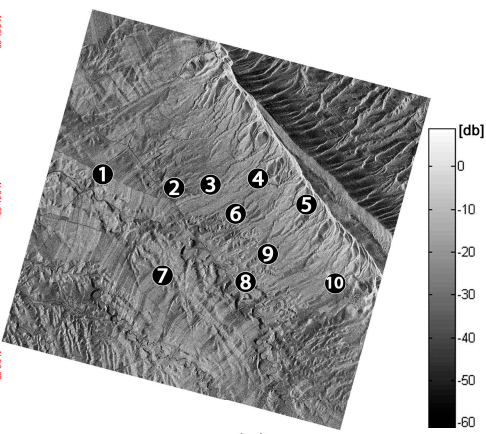

(c)

Figure 2. (a) The case study location on a map of Iran; (b) Surface roughness measurement sites position on the geological map (As, Asmari Fm; Gs, Gachsaran Fm; Pd, Pubdeh Fm; Gu, Gurpi Fm; Qu, Quaternary Sediments); (c) Measurement sites position on the X-Band SAR backscattering image; the color-bar of the values of backscattering is beside the image in $\mathrm{db}$.

Table 1. Geological age and general specification of the morphology of the study area formations.

\begin{tabular}{ccc}
\hline Geological Formation & Geological Period & General Morphology Appearance \\
\hline Asmari (As) & Oligocene to Lower Miocene & Rough \\
Gachsaran $(\mathrm{Gs})$ & early Miocene & Moderate \\
Pabdeh $(\mathrm{Pd})$ & Paleocene to early Miocene & Smooth \\
\hline
\end{tabular}

Furthermore, the SAR measured data are described in Table 2. Three SAR measurement data are used in three microwave bands. The acquisition modes are also specified.

Table 2. Specifications of the SAR measurement data in three different frequencies for the study.

\begin{tabular}{ccccc}
\hline Satellite/Antenna & Acquisition Mode & Band & Freq. (GHz) & $\begin{array}{c}\text { Incidence } \\
\text { Angle/Pass/Look }\end{array}$ \\
\hline ALOS-Palsar-2 & Spot-light & L & 1.200 & $32.3^{\circ} /$ Ascending/Left \\
Sentinel-1A & Strip Map Mode & C & 5.405 & $38.1^{\circ} /$ Ascending/Right \\
TerraSAR-X & Staring Spot-Light & X & 9.650 & $22.7^{\circ} /$ Descending/Left \\
\hline
\end{tabular}

The earth surface roughness of a single study area in each of the radar bands is seen differently, due to the difference in the operational frequency (or wavelength). Figure 3 depicts the face of the formations surface and shows the extent to which they are affected by weathering and erosion. Obviously, this variety of roughness must not be seen as the same in different wavelengths. The smoother surfaces are more susceptible to erosion, whereas the rougher ones are more resistant against weathering. The level to which a lithology gets eroded is dependent on its material and its age. 


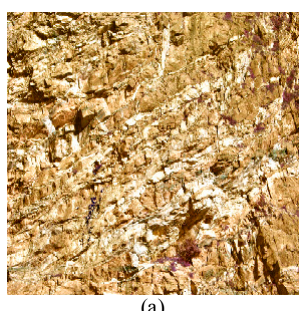

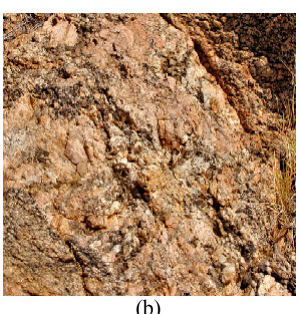

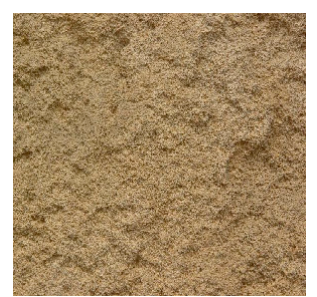

(c)

Figure 3. The geological formations surface. (a) Asmari (As) fm., Oligocene to Lower Miocene; (b) Gachsaran (Gs) fm., early Miocene; (c) Pabdeh (Pd) fm., Paleocene to early Miocene. The extent to which they are affected by weathering and erosion can be seen. The photos are taken from $\sim 1 \mathrm{~m}$ above the surface.

It must be considered that prior to any backscattering processing, as well as results assessment, the SAR imagery data introduced in Table 2, which are contaminated by the speckle noise, were subjected to a pre-processing stage, i.e., despeckling using the wavelet transform method [34].

Figure 4 illustrates how the roughness of the formations appears in the SAR images of different frequencies. The backscattering in the images is shown in the equalized black and white images, but the side color bars show the pixel values in $\mathrm{db}$. The whiter the images, the rougher the face because of double, triple, and in general, multi-scattering signals. The blacker pixel values are because of the smoothness of the surface.

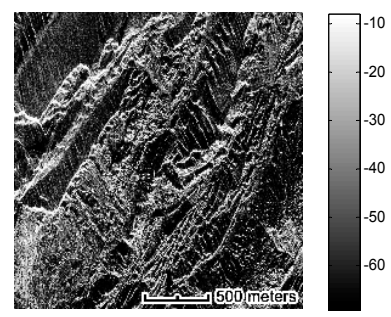

(a)

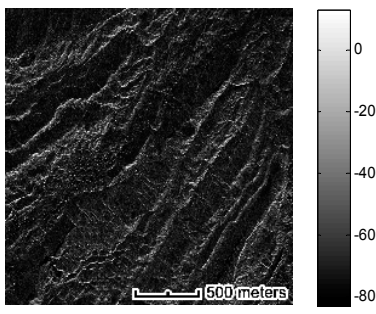

(b)

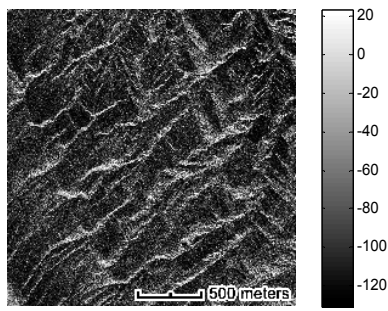

(c)

Figure 4. Backscattering on the SAR measurement, as equalized black and white images; (a) the L-Band SAR data, the ALOS-PALSAR satellite; (b) the C-Band SAR data, the Sentinel-1A satellite; (c) the X-Band SAR data, the TerraSAR-X satellite; the value of backscattering is depicted beside each image on the color-bar in $\mathrm{db}$. Lower values, i.e., dark pixels, show smoothness and large db values are because of the surface roughness.

The numerical geometric characteristics on each site surface are tabulated in Table 3. The rms-height and the correlation length are calculated using the conventional geometry; the $\alpha$ and the fractal dimension using the power-law geometry.

Since there was a considerably dry climatic condition when the satellite data was being acquired, and the field measurement was performed in the same seasonal conditions, it was obviously perceived that the moisture until $5 \mathrm{~cm}$ below the surface was absolutely $0 \%$; that is why the dielectric constant values were used from the references who presented these values for the dry climate [14]. The values for the three geological formations of the study area, i.e., Asmari, Gachsaran, and Pabdeh, are 3.6, 4.0, and 4.1 , respectively.

Figure 5 illustrates how the spectral slope $(\alpha)$ is calculated using the in situ measurements of the PSD of the 10 selected study sites. The PSDs are estimated using the Welch's power spectral density $[35,36]$. According to the previous section, the data trend or the result of large topography must not be considered in the measurement of the spectral slope $(\alpha)$. For this aim, the red line (i.e., a linear regression) in the plots of Figure 3 is drawn on a polynomial regression of the PSD, notwithstanding the general trend part of the PSD. The experimental analysis obviously shows that 
the regression of the PSD for frequencies between 0.85 and $3.15(\mathrm{~Hz})$ results in the best values for the spectral slope. This empirically decided frequency range is somehow equal for different sites.

Table 3. Morphological and surface geometric characteristics of the study sites based on the site visit and the in situ measurement. The roughness parameters are calculated using the equations and methods presented in Section 3. Figure 5 shows how $\alpha$ is estimated for each site surface. The Rms-height and the correlation length are the conventional ones and the average of the multiple arbitrary profiles on each of the site surfaces.

\begin{tabular}{ccccc}
\hline Site No. & $\begin{array}{c}\text { Dominant Geological } \\
\text { Formation }\end{array}$ & rms-Height $\mathbf{( c m )}$ & Corr. Length $(\mathbf{c m})$ & $\alpha$ \\
\hline Site 1 & As & 6.02 & 81.07 & 1.5345 \\
Site 2 & Gs & 2.2 & 45.3 & 1.7112 \\
Site 3 & As-Gs & 4.64 & 54.07 & 1.6865 \\
Site 4 & Pd & 1.11 & 14.9 & 1.7923 \\
Site 5 & Pd & 1.21 & 18.03 & 1.8825 \\
Site 6 & Gs & 1.98 & 40.71 & 1.8817 \\
Site 7 & As & 4.97 & 74.38 & 1.5349 \\
Site 8 & Gs & 1.66 & 19.31 & 1.7501 \\
Site 9 & Gs & 2.86 & 50.9 & 1.7709 \\
Site 10 & Qu & 1.76 & 31.31 & 1.7472 \\
\hline
\end{tabular}

Table 4 shows the capability of each radar frequency for the discrimination of each formation. The conformity in this table is determined according to the contents of Tables 2 and 3.

Table 4. The status of the validity of the IEM based on $(5 a, b, c)$ for each geological formation on each of the study bands, based on Fung et al. (1994) [10]. Check marks show the conformity of the formation roughness parameters (on Table 3 ) to the IEM constraints at each microwave band and the X marks show the non-conformity.

\begin{tabular}{ccccccccccc}
\hline \multirow{2}{*}{ Geological Formation } & \multicolumn{3}{c}{ L-Band } & \multicolumn{3}{c}{ C-Band } & \multicolumn{3}{c}{ X-Band } \\
\cline { 2 - 10 } & $\mathbf{5 a}$ & $\mathbf{( 5 b )}$ & $\mathbf{( 5 c )}$ & $\mathbf{( 5 a )}$ & $\mathbf{( 5 b )}$ & $\mathbf{( 5 c )}$ & $\mathbf{( 5 a )}$ & $\mathbf{( 5 b )}$ & $\mathbf{( 5 c )}$ \\
\hline Asmari Fm. & $\sqrt{ }$ & $\sqrt{ }$ & $\sqrt{ }$ & $\sqrt{ }$ & $\sqrt{ }$ & $\sqrt{ }$ & $\times$ & $\times$ & $\times$ \\
Gachsaran Fm. & $\sqrt{ }$ & $\times$ & $\sqrt{ }$ & $\sqrt{ }$ & $\sqrt{ }$ & $\sqrt{ }$ & $\sqrt{ }$ & $\times$ & $\sqrt{ }$ \\
Pabdeh Fm. & $\sqrt{ }$ & $\times$ & $\sqrt{ }$ & $\sqrt{ }$ & $\sqrt{ }$ & $\sqrt{ }$ & $\sqrt{ }$ & $\sqrt{ }$ & $\sqrt{ }$ \\
\hline
\end{tabular}

In this table, it becomes clear which of the three constraints determined by Fung et al. (1994) via $(5 a, b, c)$ are approved or rejected in order to morphologically differentiate the formations. In this table, the approbation $(\sqrt{ })$ of an equation indicates the validity of the constraint for the relevant band and the rejection $(x)$ of the equation means the invalidity of the constraint.

\subsection{Geometric Parameters Analysis}

The microwave signal, depending on the radar frequency, is sensitive to a limited range of the roughness spectrum; thus, a surface seen as being rough in one frequency measurement may be seen as completely smooth in another. Figure 6 represents the backscattering of the incident radar signal in the radar bands L, C, and X on $h h, v v$, and $h v$ polarizations for the earth surface of the main geological formations present in the field measured sites. As the frequency or the local incident angle increases, the smaller roughness scale becomes significant. The graphs confirm the different capability of the operational frequencies for the surfaces discrimination, presented in Table 4. 

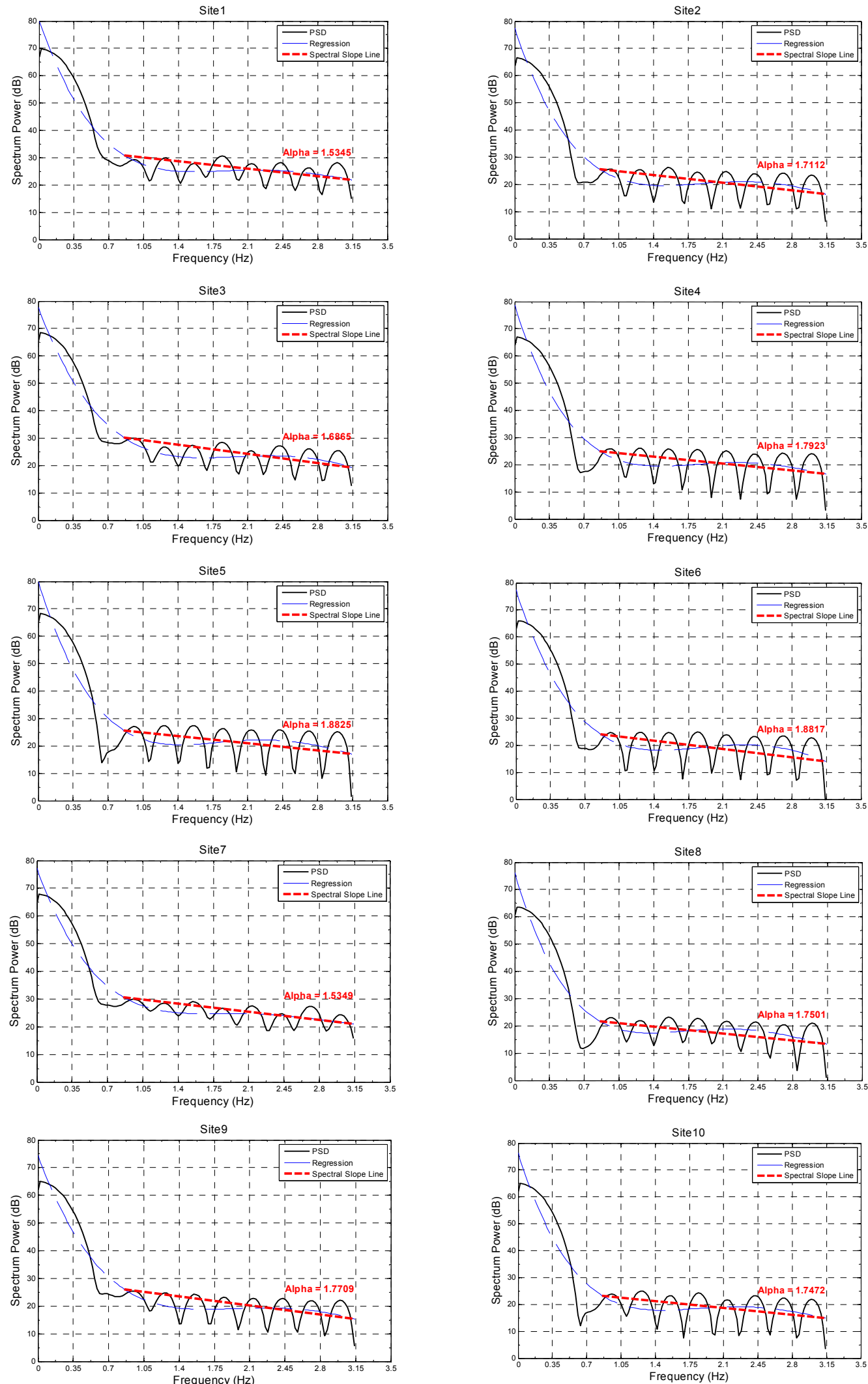

Figure 5. Calculation of the spectral slope $(\alpha)$ via the linear regression of a polynomial regression of the PSD, calculated from the in situ measurements of the 10 selected study sites using the Welch's method. Experimentally, the trend of the data is between 0 and $0.85 \mathrm{~Hz}$, which has not been considered in the calculation of the spectral slope. 

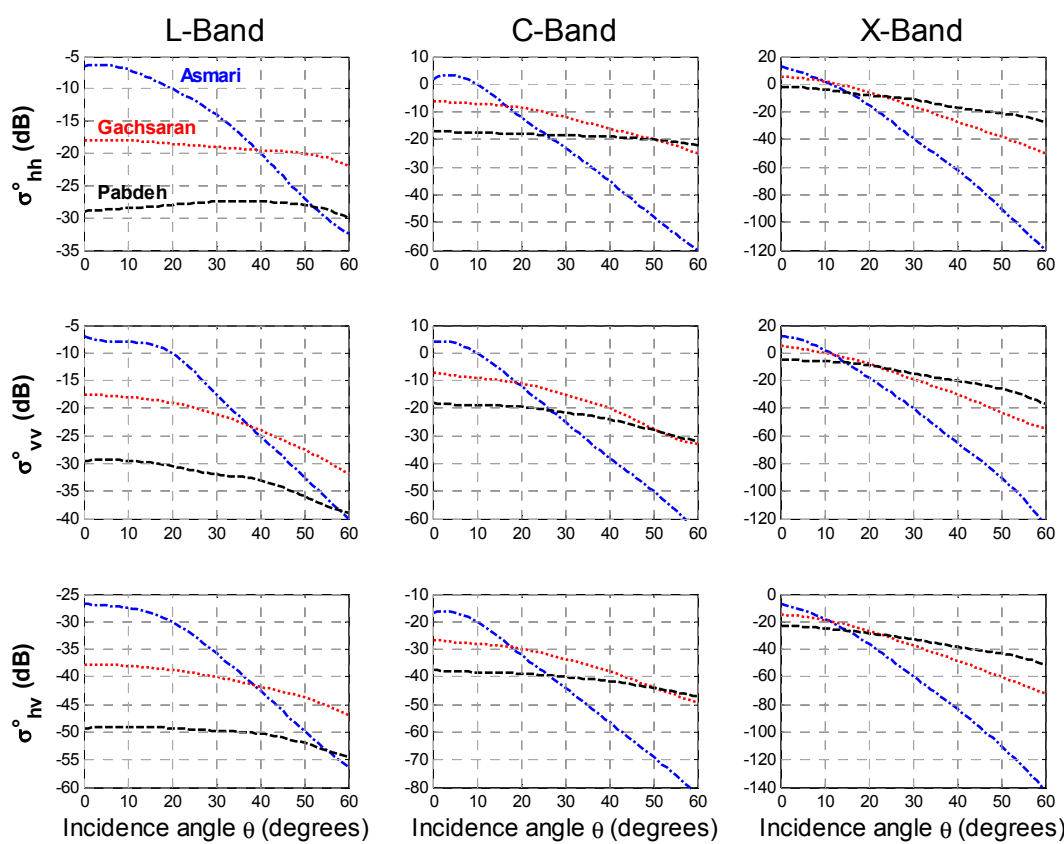

Figure 6. Backscattering $\left(\sigma^{\circ}\right)$ for three bands $\mathrm{L}, \mathrm{C}$, and $\mathrm{X}$ on $h h, v v$, and $h v$ polarizations for the main geological formations of the study area.

\subsection{Evaluation of Backscattering and Surface Roughness Simulation}

What is needed to discriminate geological formations according to their morphology is the surface roughness [25]. In this section, the results of the IEM implementation to simulate the backscattering coefficient $\left(\sigma^{\circ}\right)$ using the surface data and a comparison with the SAR measured ones in the radar bands $\mathrm{L}, \mathrm{C}$, and $\mathrm{X}$ for the surface roughness computation is described (Figure 1a). Via inverse IEM using the LUT, having ascertained the SAR measurements, the rms-height of the surface is simulated. An evaluation of the simulated surface roughness can be performed using the rms-height of the field measurements (Figure 1b).

Having obtained the surface geometric characteristics for a $25 \times 25$ points mesh and then, taking arbitrary profiles according to geological maps and the site visit, a matrix $25 \times 25$ of $\sigma^{\circ}$ is computed for each site. Moreover, using the IEM inversion, by the values of $\sigma^{\circ}$ on a $25 \times 25$ pixels SAR image, a $25 \times 25$ matrix of the rms-height can be calculated for each of the sites.

The statistical rms-height, as well as the ACF, are calculated beside the extracted dielectric constant of each site from the published measured values to compute the conventional IEM backscattering coefficient, i.e., the values of $\sigma_{h h}^{\circ}$ and $\sigma_{v v}^{\circ}$. The power-law IEM is computed using the ACF and the rms-height is calculated using the spectral slope parameter.

Figures 7-9, respectively, for the bands L, C, and X, present the comparisons between the backscattering coefficients calculated through the three methods of calculation of the IEM input parameters, namely two "Conventional" methods (exponential and Gaussian ACFs) and a "Power-law" method, in comparison with the measured SAR backscattering coefficient, referred to as an "SAR Measurement". This comparison is performed on 30 randomly selected pixels for 10 measured sites. Precisely being a point on the diagonal line of each graph indicates that the IEM simulated value on the corresponding pixel is exactly equal to the measured backscattering on that pixel. Therefore, in these graphs, the farness of the diagonal line shows the simulation error [25,37]. 

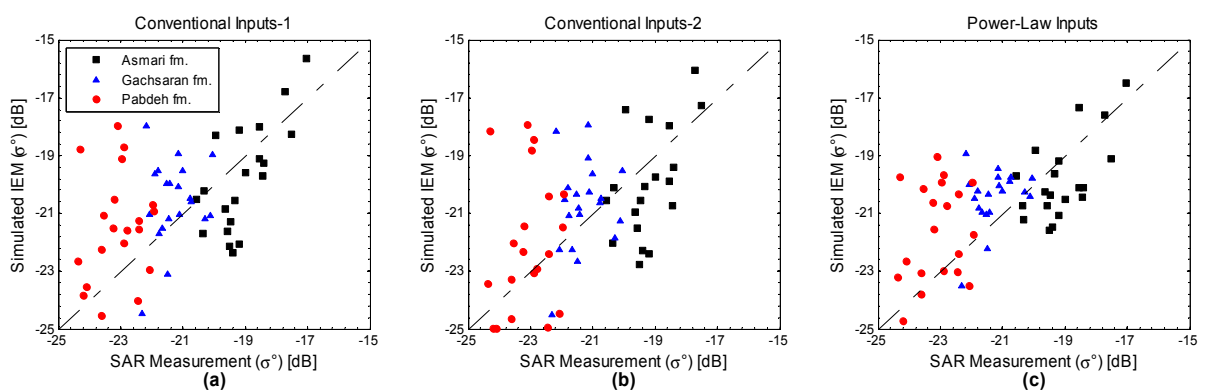

Figure 7. Backscattering simulation accuracy via the IEM for the geological formations on the L-band, (a) using the conventional geometry and the exponential ACF; (b) using the conventional geometry and the Gaussian ACF; (c) using the Power-Law geometry for the calculation of the IEM input parameters.
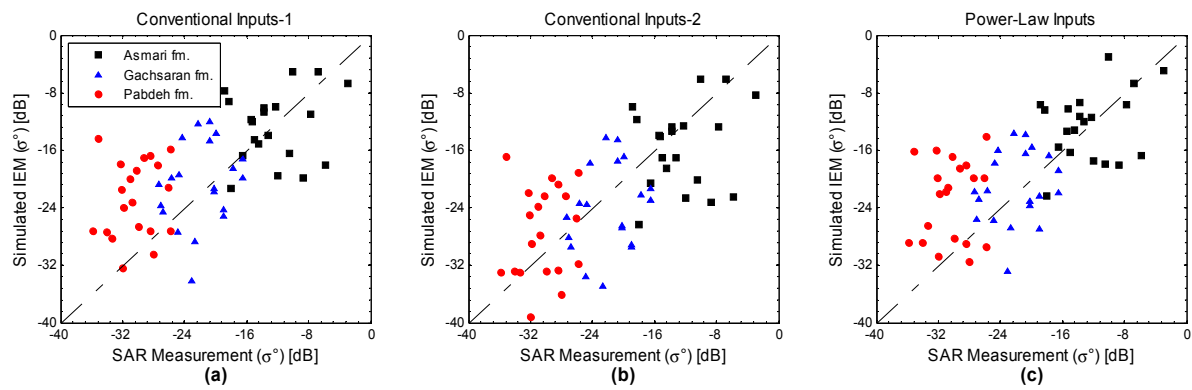

Figure 8. Backscattering simulation accuracy via the IEM for the geological formations on the C-band, (a) using the conventional geometry and the exponential ACF; (b) using the conventional geometry and the Gaussian ACF; (c) using the Power-Law geometry for the calculation of the IEM input parameters.

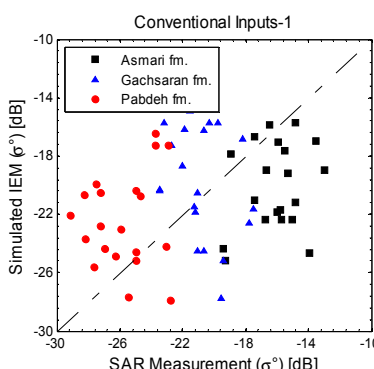

(a)

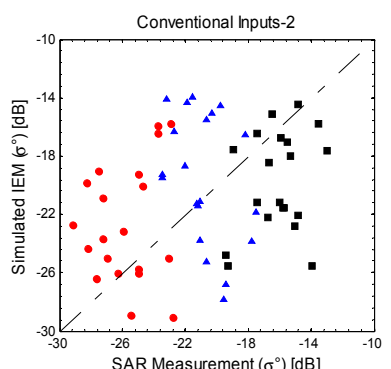

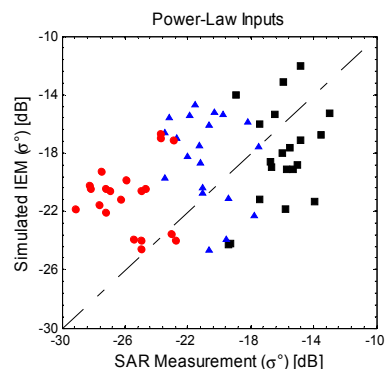

(c)

Figure 9. Backscattering simulation accuracy via the IEM for the geological formations on the X-band, (a) using the conventional geometry and the exponential ACF; (b) using the conventional geometry and the Gaussian ACF; (c) using the Power-Law geometry for the calculation of the IEM input parameters.

For an assessment of the aforementioned calculation methods, it is necessary to compare the simulated surface roughness parameter (rms-height) resulting from the inverse IEM (i.e., extracted from Look-up table) with the in situ measured parameter for the ten sites. For this comparison, the point graphs of Figures 10-12 depict the comparison of the computed rms-height of 30 selected pixels on bands L, C, and X, and the measured values on their corresponding field points.

Precisely being a point on the diagonal line of each graph indicates that the simulated rms-height using the inverse IEM on the corresponding pixel is exactly equal to the field measured rms-height. Evidently, the proximity of the points to the diagonal line announces the simulation correctness [37]. 

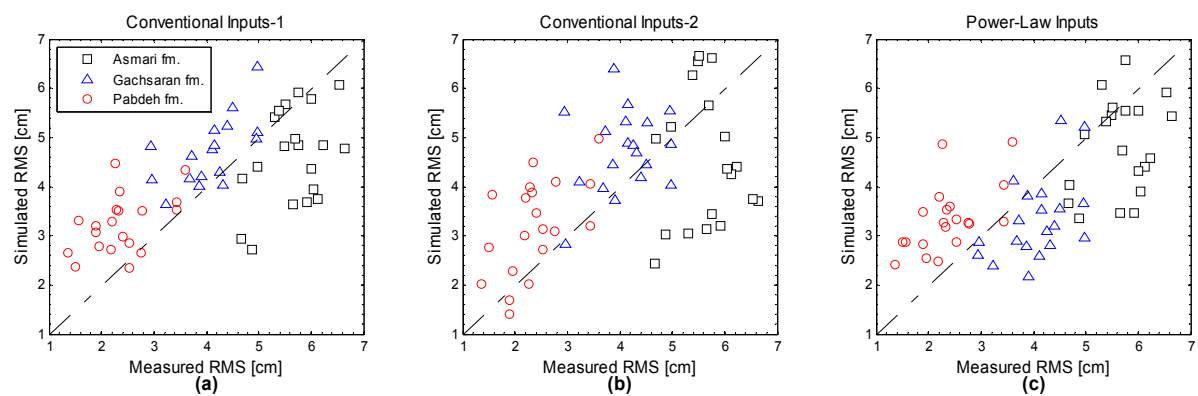

Figure 10. The Rms-height simulation accuracy via the inverse IEM for the geological formations on the L-band, (a) Using the conventional geometry and the exponential ACF; (b) using the conventional geometry and the Gaussian ACF; (c) using the Power-Law geometry for the calculation of the IEM input parameters.

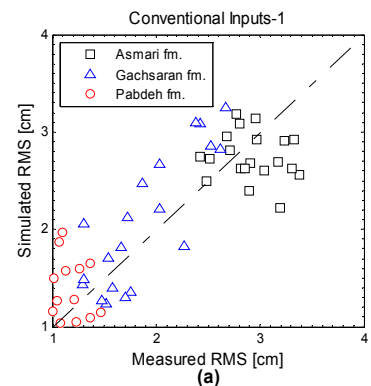

(a)

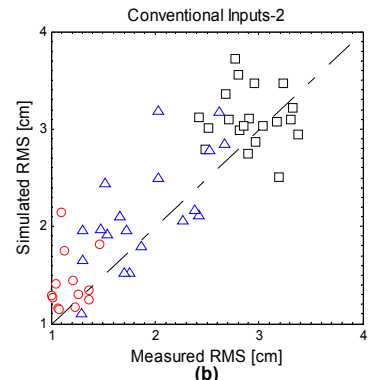

(b)

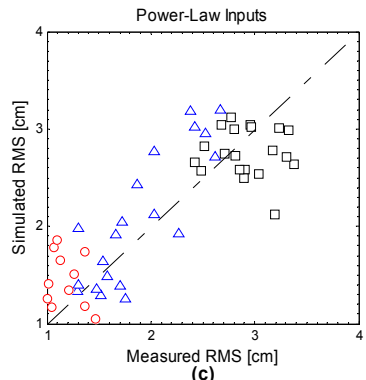

(c)

Figure 11. The Rms-height simulation accuracy via the inverse IEM for the geological formations on the C-band, (a) Using the conventional geometry and the exponential ACF; (b) using the conventional geometry and the Gaussian ACF; (c) using the Power-Law geometry for the calculation of the IEM input parameters.

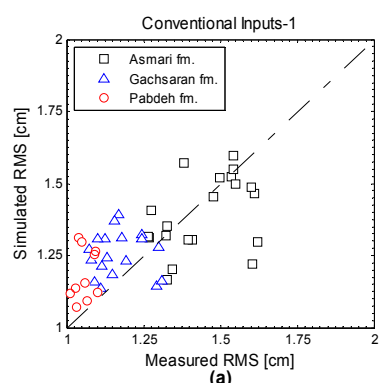

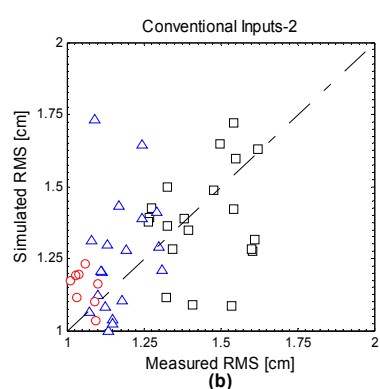

(b)

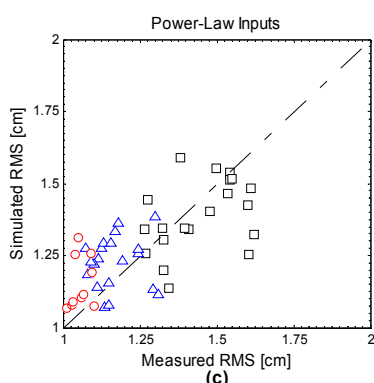

(c)

Figure 12. The Rms-height simulation accuracy via the inverse IEM for the geological formations on the X-band, (a) using the conventional geometry and the exponential ACF; (b) using the conventional geometry and the Gaussian ACF; (c) using the Power-Law geometry for the calculation of the IEM input parameters.

In Table 5, the standard deviation of the calculated rms-height in the methods of IEM implementation are tabulated. Therefore, the statistical dispersion of the calculated results on each site for each polarization versus the field measured values are comparable, in order to evaluate the efficiency of the proposed power-law calculation of the inputs instead of the conventional one. Evidently, the smaller the standard deviation, the higher the accuracy of the simulated data. 
Table 5. The standard deviation of the rms-height, calculated using the inverse IEM for the geological formations on bands L, C, and X, using three methods of inputs calculation, two conventional (1: The exponential ACF; 2: The Gaussian ACF) and the power-law geometry. The bold values show a decrease of the standard deviation of using the power-law inputs versus both the conventional ones.

\begin{tabular}{ccccccccccc}
\hline \multirow{2}{*}{ Geological Formation } & \multicolumn{3}{c}{ L-Band } & \multicolumn{3}{c}{ C-Band } & \multicolumn{3}{c}{ X-Band } \\
\cline { 2 - 10 } & As. & Gs. & Pd. & As. & Gs. & Pd. & As. & Gs. & Pd. \\
\hline Inputs using the Conventional Geometry1 & 1.049 & 0.919 & 0.873 & 0.352 & 0.378 & 0.505 & 0.207 & 0.239 & 0.341 \\
Inputs using the Conventional Geometry2 & 0.985 & 0.799 & 0.834 & 0.375 & 0.309 & 0.506 & 0.232 & 0.283 & 0.355 \\
Inputs using the Power-law Geometry & $\mathbf{0 . 8 3 9}$ & $\mathbf{0 . 7 2 1}$ & 0.970 & $\mathbf{0 . 3 2 6}$ & 0.370 & 0.512 & 0.224 & 0.240 & $\mathbf{0 . 2 8 5}$ \\
\hline
\end{tabular}

Comparing the values of the standard deviation in Table 5 shows that the use of random fractals geometry has improved the results by more than $10 \%$ on average, i.e., dividing the largest standard deviation values of the two conventional methods by the standard deviation of the Power-law method can give such a value. The bold numbers are used to indicate that the Power-law geometry overcomes both of the conventional methods. For both co-polarizations, the same improvement is achieved.

Figure 13 illustrates the standard deviation values on the bar-charts to easily compare the different frequencies, as well as the different formations.

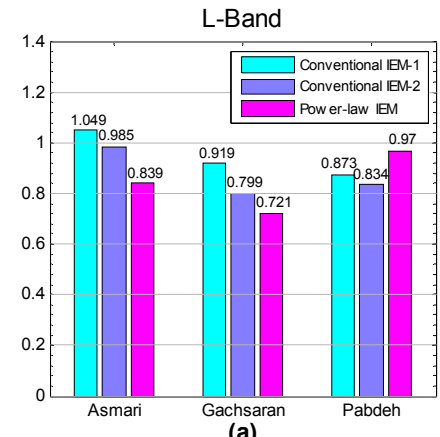

(a)

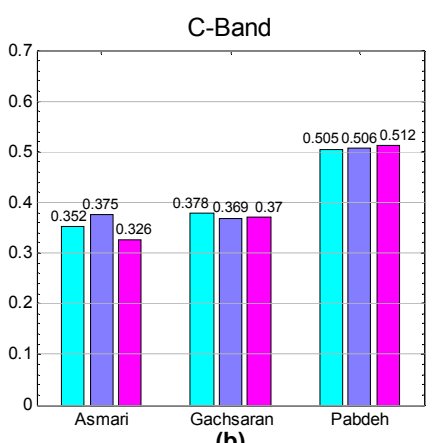

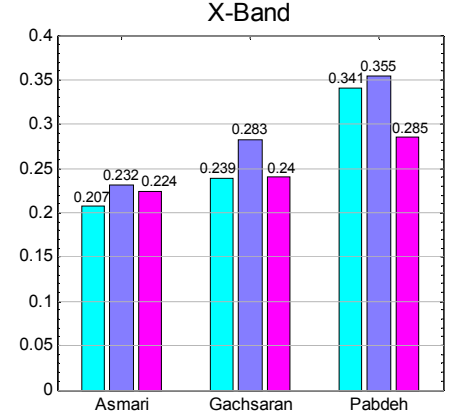

(c)

Figure 13. The Standard Deviation of the calculated rms-height values using the IEM Look-up table calculated via three methods of inputs calculation, conventional-1: using the exponential ACF, the conventional-2: using the Gaussian ACF, and the power-law geometry for the geological formations of the study sites, using the backscattering coefficient on (a) L-band; (b) C-band; and (c) X-band.

\section{Discussion}

In this paper, the Integral Equation Model (IEM) backscattering model, as well as its inverse (using the Look-up table method), are implemented to measure the geometry of the earth surface roughness or morphology. For such implementation, the input parameters are calculated using three different methods: two conventional geometries (the exponential and the Gaussian correlation functions, the ACFs) and one power-law method. The implementation of the fractal geometry in this paper is performed through the spectral slope parameter $(\alpha)$, which shows the coincidence of the power-law ACF with the earth surface. The IEM results (i.e., $\sigma^{\circ}$, backscattering coefficients) are compared with the Synthetic Aperture Radar (SAR) measurement (i.e., TerraSAR-X) and the inverse IEM results (i.e., rms-height) were compared with the in situ measurement.

The fractal nature of the roughness causes a higher efficiency of the power-law geometry versus the conventional geometry when modeling the surface; Figures 7-12 show better results for the fractal modeling and eliminate more uncertainties, affirming $[21,38]$ studies. Comparing the two conventional geometry implementations, apart from the C-band as the moderate wavelength, the Gaussian ACF causes less deviation for higher wavelengths, and the exponential ACF results are better for lower wavelengths; this supports the deductions presented in $[12,37,39]$. The values of the standard deviation in Table 5 show a general improvement, but when considering the behavior of different geological 
surfaces with various levels of roughness on each of the SAR frequency image, we deduce that for lower frequencies (e.g., L-band), the rougher surfaces are in the fractal regime, confirming the conclusions presented in [21,40]; and for the higher frequencies (e.g., X-band), the smoother ones are in accordance with [6]. Therefore, the rougher surfaces in high frequencies and the smoother ones in low frequencies display an obvious diffractal behavior. In general, having the in situ measurements, applying the most famous backscattering model, and completing an evaluation with the SAR measured data, the results of significant studies such as $[17,38,41,42]$ are somehow endorsed with comparable numerical results, showing the efficiency of applying the power-law methods for IEM implementation for the SAR backscattering studies.

\section{Conclusions}

Using the random fractal geometry via the implementation of the power-law model in the backscattering modeling offers results which are up to $10 \%$ better for the calculation of the surface geometry. This methodology can improve the results of the spectral processings in the morphological mapping of the formations. Additionally, using the achieved information of morphology, we improved the scale of the geological maps.

For each radar frequency, the specific size of the surface roughness can be measured via signal backscattering. Using the three SAR bands L, C, and X for the earth surface of three known-geological surfaces shows the capability of this technology in geological morphology mapping.

The selection of the sites in this paper was undertaken considering that no large-scale topography has been inside the sampling mesh. Moreover, for the calculation of the spectral slope parameter $(\alpha)$, the trend part of the Power Spectral Density (PSD) graph has been ignored, and the spectral slope line was drawn on a polynomial regression of the PSD, which experimentally offered more accurate results.

A proper estimation of the surface roughness spatial frequency and consequently the spectral slope $\alpha$, using the power-law ACF, offered appropriate IEM input parameters computation; therefore, after running the model, a more precise backscattering coefficient which exhibited a lower standard deviation was achieved. Also, using the random fractal geometry, the model inversion with the Look-up table method offered better roughness approximation. However, the geometric behavior of the surface roughness against the SAR frequencies is not constant, i.e., as the micro-topography decreases, the fractal regim exists in higher frequencies, and vice versa.

Acknowledgments: The authors would like to thank the University of Tehran Vice Chancellor for the Research and also the Research and Development department of NIOC Exploration Directorate for the support of this paper. All the respectable reviewers are acknowledged for their fruitful comments and suggestions about the paper.

Author Contributions: A.G., J.A., M.D., and M.A.K. contributed to conception. A.G. and M.A.K. carried out the acquisition, analysis, and interpretation of data. A.G. drafted the manuscript. All authors read and approved the final manuscript.

Conflicts of Interest: The authors declare that they have no competing interests.

\section{References}

1. Panciera, R.; Tanase, M.A.; Lowell, K.; Walker, J.P. Evaluation of iem, dubois, and oh radar backscatter models using airborne l-band sar. IEEE Trans. Geosci. Remote Sens. 2014, 52, 4966-4979. [CrossRef]

2. Rodrigues, F.Á.; Neto, J.R.; Marques, R.P.; de Medeiros, F.S.; Nobre, J.S. Sar image segmentation using the roughness information. IEEE Geosci. Remote Sens. Lett. 2016, 13, 132-136. [CrossRef]

3. Zhu, L.; Walker, J.P.; Ye, N.; Rudiger, C. The effect of radar configuration on effective correlation length. In Proceedings of the 2016 International Conference on Electromagnetics in Advanced Applications (ICEAA), Cairns, Australia, 19-23 September 2016; pp. 820-823.

4. Baghdadi, N.; Zribi, M.; Paloscia, S.; Verhoest, N.E.; Lievens, H.; Baup, F.; Mattia, F. Semi-empirical calibration of the integral equation model for co-polarized l-band backscattering. Remote Sens. 2015, 7, 13626-13640. [CrossRef] 
5. Gorrab, A.; Zribi, M.; Baghdadi, N.; Mougenot, B.; Chabaane, Z.L. Potential of x-band terrasar-x and cosmo-skymed sar data for the assessment of physical soil parameters. Remote Sens. 2015, 7, 747-766. [CrossRef]

6. Martínez-Agirre, A.; Álvarez-Mozos, J.; Lievens, H.; Verhoest, N.E.; Giménez, R. Sensitivity of c-band backscatter to surface roughness parameters measured at different scales. In Proceedings of the 2015 IEEE International Geoscience and Remote Sensing Symposium (IGARSS), Milan, Italy, 26-31 July 2015; pp. 700-703.

7. Ulaby, F.T.; Long, D.G. Microwave Radar and Radiometric Remote Sensing; University of Michigan Press: Ann Arbor, MI, USA, 2014; pp. 425-445.

8. Baghdadi, N.; Cresson, R.; Pottier, E.; Aubert, M.; Mehrez, M.; Jacome, A.; Benabdallah, S. A potential use for the c-band polarimetric sar parameters to characterize the soil surface over bare agriculture fields. IEEE Trans. Geosci. Remote Sens. 2012, 50, 3844-3858. [CrossRef]

9. Zribi, M.; Gorrab, A.; Baghdadi, N. A new soil roughness parameter for the modelling of radar backscattering over bare soil. Remote Sens. Environ. 2014, 152, 62-73. [CrossRef]

10. Fung, A.K. Microwave Scattering and Emission Models and Their Applications; Artech House: Norwood, MA, USA, 1994.

11. Fung, A.K.; Li, Z.; Chen, K. Backscattering from a randomly rough dielectric surface. IEEE Trans. Geosci. Remote Sens. 1992, 30, 356-369. [CrossRef]

12. Hajnsek, I. Inversion of Surface Parameters Using Polarimetric Sar. Ph.D. Thesis, Friedrich-SchillerUniversität Jena, Jena, Germany, 2001.

13. Franceschetti, G.; Riccio, D. Scattering, Natural Surfaces, and Fractals; Academic Press: Cambridge, MA, USA, 2006.

14. Martinez, A.; Byrnes, A.P. Modeling Dielectric-Constant Values of Geologic Materials: An Aid to Ground-Penetrating Radar Data Collection and Interpretation; Kansas Geological Survey, University of Kansas: Lawrence, KS, USA, 2001.

15. Mazaheri Tehrani, H. Soil Moisture Estimation with Polarimetric Sar Data. Ph.D. Thesis, University of Calgary, Calgary, AL, Canada, 2014.

16. Fung, A.K.; Chen, K.S. An update on the iem surface backscattering model. IEEE Geosci. Remote Sens. Lett. 2004, 1, 75-77. [CrossRef]

17. Baghdadi, N.; Holah, N.; Zribi, M. Calibration of the integral equation model for sar data in c-band and hh and vv polarizations. Int. J. Remote Sens. 2006, 27, 805-816. [CrossRef]

18. Barrett, B.W.; Dwyer, E.; Whelan, P. Soil moisture retrieval from active spaceborne microwave observations: An evaluation of current techniques. Remote Sens. 2009, 1, 210-242. [CrossRef]

19. Chen, K.; Kao, W.; Tzeng, Y. Retrieval of surface parameters using dynamic learning neural network. Remote Sens. 1995, 16, 801-809. [CrossRef]

20. Sahebi, M.R.; Bonn, F.; Bénié, G.B. Neural networks for the inversion of soil surface parameters from synthetic aperture radar satellite data. Can. J. Civ. Eng. 2004, 31, 95-108. [CrossRef]

21. Dierking, W. Quantitative roughness characterization of geological surfaces and implications for radar signature analysis. IEEE Trans. Geosci. Remote Sens. 1999, 37, 2397-2412. [CrossRef]

22. Álvarez-Mozos, J.; Verhoest, N.E.; Larrañaga, A.; Casalí, J.; González-Audícana, M. Influence of surface roughness spatial variability and temporal dynamics on the retrieval of soil moisture from sar observations. Sensors 2009, 9, 463-489. [CrossRef] [PubMed]

23. Choker, M.; Baghdadi, N.; Zribi, M.; El Hajj, M.; Paloscia, S.; Verhoest, N.E.; Lievens, H.; Mattia, F. Evaluation of the oh, dubois and iem backscatter models using a large dataset of sar data and experimental soil measurements. Water 2017, 9, 38. [CrossRef]

24. Verhoest, N.E.; Lievens, H.; Wagner, W.; Álvarez-Mozos, J.; Moran, M.S.; Mattia, F. On the soil roughness parameterization problem in soil moisture retrieval of bare surfaces from synthetic aperture radar. Sensors 2008, 8, 4213-4248. [CrossRef] [PubMed]

25. Ghafouri, A.; Amini, J.; Dehmollaian, M.; Kavoosi, M. Measuring surface roughness of geological rock surfaces in sar data using fractal geometry. C. R. Geosci. 2017, 11, 327-338.

26. Baghdadi, N.; Chaaya, J.A.; Zribi, M. Semiempirical calibration of the integral equation model for sar data in c-band and cross polarization using radar images and field measurements. IEEE Geosci. Remote Sens. Lett. 2011, 8, 14-18. [CrossRef] 
27. Western, A.W.; Bloschl, G.; Grayson, R.B. How well do indicator variograms capture the spatial connectivity of soil moisture? Hydrol. Process. 1998, 12, 1851-1868. [CrossRef]

28. Mandelbrot, B.B. The Fractal Geometry of Nature; Macmillan Publishers: London, UK, 1983.

29. Durst, P.J.; Mason, G.L.; McKinley, B.; Baylot, A. Predicting rms surface roughness using fractal dimension and psd parameters. J. Terramech. 2011, 48, 105-111. [CrossRef]

30. Martino, G.D.; Franceschetti, G.; Riccio, D.; Zinno, I. Spectral processing for the extraction of fractal parameters from sar data. In Proceedings of the 2011 17th International Conference on Digital Signal Processing (DSP), Corfu, Greece, 6-8 July 2011; pp. 1-7.

31. Yordanov, O.; Atanasov, I. Self-affine random surfaces. Eur. Phys. J. B 2002, 29, 211-215. [CrossRef]

32. Yordanov, O.; Ivanova, K. Description of surface roughness as an approximate self-affine random structure. Surf. Sci. 1995, 331, 1043-1049. [CrossRef]

33. Rasigni, G.; Llebaria, A.; Lafraxo, M.; Buat, V.; Rasigni, M.; Abdellani, F. Autoregressive process for characterizing statistically rough surfaces. J. Opt. Soc. Am. A 1993, 10, 1257-1262. [CrossRef]

34. Roomi, S.; Kalaiyarasi, D.; Rangan, N.K. Discrete wavelet transform based despeckling for sar images. In Proceedings of the 2011 World Congress on Information and Communication Technologies, Mumbai, India, 11-14 December 2011; pp. 373-378.

35. Wang, F.; Zhang, B.; Yang, D.; Li, W.; Zhu, Y. Sea-state observation using reflected beidou geo signals in frequency domain. IEEE Geosci. Remote Sens. Lett. 2016, 13, 1656-1660. [CrossRef]

36. Fadzal, C.C.W.; Mansor, W.; Khuan, L.; Mohamad, N.; Mahmoodin, Z.; Mohamad, S.; Amirin, S. Welch power spectral density of eeg signal generated from dyslexic children. In Proceedings of the 2014 IEEE Region 10 Symposium, Kuala Lumpur, Malaysia, 14-16 April 2014; pp. 560-562.

37. Ghafouri, A.; Amini, J.; Dehmollaian, M.; Kavoosi, M. Random fractals geometry in surface roughness modeling of geological formations using synthetic aperture radar images. J. Geomat. Sci. Technol. Iran. Soc. Surv. Geomat. Eng. 2015, 5, 97-108.

38. Di Martino, G.; Iodice, A.; Riccio, D.; Ruello, G.; Zinno, I. On the fractal nature of volcano morphology detected via sar image analysis: The case of somma-vesuvius volcanic complex. Eur. J. Remote Sens. 2012, 45, 177-187. [CrossRef]

39. Zribi, M. Développement de Nouvelles Méthodes de Modélisation de la Rugosité Pour la Rétrodiffusion Hyperfréquence de la Surface Du Sol. Ph.D. Thesis, Université Paul Sabatier, Toulouse, France, 1998.

40. Franceschetti, G.; Iodice, A.; Maddaluno, S.; Riccio, D. A fractal-based theoretical framework for retrieval of surface parameters from electromagnetic backscattering data. IEEE Trans. Geosci. Remote Sens. 2000, 38, 641-650. [CrossRef]

41. Franceschetti, G.; Iodice, A.; Migliaccio, M.; Riccio, D. Scattering from natural rough surfaces modeled by fractional brownian motion two-dimensional processes. IEEE Trans. Antennas Propag. 1999, 47, 1405-1415. [CrossRef]

42. Zribi, M.; Ciarletti, V.; Taconet, O.; Paillé, J.; Boissard, P. Characterisation of the soil structure and microwave backscattering based on numerical three-dimensional surface representation: Analysis with a fractional brownian model. Remote Sens. Environ. 2000, 72, 159-169. [CrossRef]

(C) 2017 by the authors. Licensee MDPI, Basel, Switzerland. This article is an open access article distributed under the terms and conditions of the Creative Commons Attribution (CC BY) license (http:/ / creativecommons.org/licenses/by/4.0/). 